\title{
Prospective Study of Correlation between Carotid Intimal Medial Thickness and Non Alcoholic Fatty Liver Disease Along with Its Grades
}

\author{
Dr. Darshana Verma (Md General Medicine) ${ }^{1}$, Dr. Vinayak Gour (Md Anaesthesia) ${ }^{2}$
}

\begin{abstract}
This study was performed to study correlation between CIMT and NAFLD. This was hospital-based prospective study conducted at the Department of Medicine, Hamidia Hospital and Gandhi Medical College, Bhopal during period of May 2014 to Nov 2014. 100 patients with ultrasonographically proven NAFLD patients from OPD and IPD(45 males and 55 females $) A N D$ 100 patients (47 males 53 males) without NAFLD on ultrasonography abdomen were studied. The grade of NAFLD was also taken into correlation for CIMT thickness. After obtaining informed written consent, the general information of patients was recorded on a proforma. After overnight fasting, blood samples was drawn for complete hemogram, blood urea, serum creatinine, lipid profile and serum Mg. Carotid intima media thickness (CIMT) was measured by B mode ultrasound using a 7.5MHz transducer. Descriptive and inferential statistical analyses were performed using SPSS version 20.0.the study shows that average age in NAFLD group was 77.7yrs and was higher than in control group that was 56.33yrs.The study shows that mean BMI is higher in subjects with NAFLD in both males and females as compared to that in non NAFLD group. The study shows that mean Waist/hip ratio is higher in subjects with NAFLD in both males and females as compared to that in non NAFLD group. Serum SGOT and SGPT levels were found to be significantly higher in subjects with NAFLD as compared to that in non NAFLD group.The study shows that CIMT on both sides were higher on NAFLD group as compared to non NAFLD group. We concluded that patients with NAFLD have significant positive correlation, and CIMT (significantly correlated positively with grades of NAFLD as well).It is suggested that since NAFLD along with the grades is associated with inflammation of liver, metabolicsyndrome, increased CIMT(which is a proven marker for atherosclerosis an d a predictor of CAD and CVA).so now with further research and analysis NAFLD could also be considered as a marker for atherosclerosis and as extended spectrum of metabolic syndrome.
\end{abstract}

Keywords: NAFLD non alcoholic fatty liver disease, CIMT- carotid intima medial thickness

\section{Introduction}

Non-alcoholic fatty liver disease (NAFLD) is a highly prevalent condition which is characterized by fatty infiltration of liver cells resembling that of alcohol-induced liver injury but occuring in patients who do not abuse alcohol [1]. The incidence of fatty liver is estimated at 14$23 \%$. This figure is $70-90 \%$ in obese and type II diabetic people [2]. The prevalence of NAFLD is $20 \%-30 \%$ in the general adult population in Western countries(123) and is now $12 \%-24 \%$ in Asian-Pacific countries.(14) Its prevalence in obese or diabetic patients increases up to $70 \%-90 \%$.(12) The spectrum of NAFLD ranges from fatty liver alone to steatohepatitis, which histologically is similar to alcoholic hepatitis, and may progress to end-stage liver disease and cirrhosis. That is why the fatty liver, long considered an incidental finding, has received increasing attention [3]. The previous studies have shown that ultrasonographic results are well correlated with histological findings due to fatty infiltration $[4,5]$. Therefore, ultrasonography alone can indicate the level of fatty infiltration in liver. On the other hand, NAFLD is strongly associated with obesity, type II diabetes, and dyslipidemia. In addition, the majority of these patients have central obesity and insulin resistance symptoms. Consequently, NAFLD shares many features of the metabolic syndrome, which is a highly atherogenic condition, and its presence is associated with marked cardiovascular risk [1]. It has been shown that intima-media thickness (IMT) of carotid artery, as a reliable index of subclinical atherosclerosis, is a predictor of myocardial infarction and stroke. non- alcoholic steatohepatitis (NASH) represents the more advanced stages of this disease, i.e. the 'inflammatory' component in addition to steatosis, which carries a higher risk of $\mathrm{CV}$ disease and mortality than simple steatosis. Insulin resistance and obesity, both key features of the MetS, are strongly associated with NAFLD progression

\section{Definition of non-alcoholic fatty liver disease}

Non-alcoholic fatty liver disease is the most common cause of chronic liver disease in the general population and is present when fatty infiltration affects $.5 \%$ of hepatocytes, in the presence of , <20 g (2.5 U) of alcohol consumption per day, without evidence of other causes of liver disease

\section{Diagnosis of non-alcoholic fatty liver disease}

up to $70 \%$ of NAFLD patients may have normal liver enzymes and although alanineaminotransferase (ALT) levels have shown to be the best single biochemical correlate of hepatic steatosis, they do not distinguish between varying stages of NASH and can be normal in histologically severe disease . Furthermore, ultrasound imaging can only detect steatosis when $.30 \%$ of the liver is affected, but is still recommended as the first-line investigation to 'confirm' the presence of fatty liver due to its wide spread availability and low cost, none of the non-invasive modalities can detect inflammation and/or fibrosis, i.e. NASH. Consequently, liver biopsy is at present the 'gold-standard' (taking into account potential inaccuracies of sampling variability) for diagnosing NAFLD and staging the degree of NASH and fibrosis by histological assessment, as well as monitoring disease progression 


\section{International Journal of Science and Research (IJSR) ISSN (Online): 2319-7064 \\ Index Copernicus Value (2013): 6.14 | Impact Factor (2014): 5.611}

\section{Methods}

100 patients with diagnosis of NAFLD and 100 patients without NAFLDattendingopd or ipd to medicine department of Hamidia Hospital and GMC Bhopal were taken for the study from aPeriod of collection of data: MAY2013 -DEC 2014.The patient excluded were 1)those with a history of known liver disease, HepB ,Hep C positivity. 2) those who consume more than $20 \mathrm{~g}$ alcohol daily or abuse alcohol, and 3 ) those with ultrasonic-proven liver disease other than fatty liver.4) those with other known co-morbid conditions like hypothyroidism, History of carotid surgery, Malignancy, Significant illness.Information was collected through prepared proforma for each patient and informed consent was obtained from each participant. The study protocol was approved by Institutional Ethics Committee of Gandhi medical college, Bhopal.History of smoking, DM, hypertension. Cardiovascular event, hyperlipidemia were evaluated. A complete clinical examination was done. Hypertension was defined as blood pressure $>140 / 90 \mathrm{~mm}$ $\mathrm{Hg}$ or if patient is already on antihypertensive drug. The morning urine sample and blood samples were collected after 8 hours of overnight fasting for completehemogram, blood urea levels, serum creatinine levels,,FBS, serum elecrolytes and lipid profile (Total cholesterol, Triglycerides and HDL). All the biochemical parameters were measured by standard laboratory technique.Carotid intima media thickness was measured by B mode ultrasound using a $7.5 \mathrm{MHz}$ transducer. Intima Media Thickness was defined as distance between leading edge of first echogenic line (Lumen - Intima interface) and second echogenic line
(Media - Adventitia interface) of far wall. Three measurements were taken $0.5,1$ and $2 \mathrm{~cm}$ below carotid bifurcation of common carotid artery on each side. The arithmetical averages of these were taken. The IMT of both sides (right and left) was calculated CIMT measurement was always performed by single radiologist in plaque free arterial segments.

\section{Observations}

\begin{tabular}{|c|c|c|c|}
\hline NAFLD & CIMT thick & Normal & Total \\
\hline \multirow{2}{*}{ gr 1} & 36 & 27 & 63 \\
\cline { 2 - 4 } & $57.1 \%$ & $42.9 \%$ & $100.0 \%$ \\
\hline \multirow{2}{*}{ gr2 } & 33 & 0 & 33 \\
\cline { 2 - 4 } & $100.0 \%$ & $0.0 \%$ & $100.0 \%$ \\
\hline \multirow{2}{*}{ gr3 } & 4 & 0 & 4 \\
\cline { 2 - 4 } & $100.0 \%$ & $0.0 \%$ & $100.0 \%$ \\
\hline \multirow{2}{*}{ Total } & 73 & 27 & 100 \\
\cline { 2 - 4 } & $73.0 \%$ & $27.0 \%$ & $100.0 \%$ \\
\hline
\end{tabular}

\begin{tabular}{|c|c|c|c|c|}
\hline & $\begin{array}{c}\text { NAFLD } \\
\text { gr1 }\end{array}$ & $\begin{array}{c}\text { NAFLD } \\
\text { gr2 }\end{array}$ & $\begin{array}{c}\text { NAFLD } \\
\text { gr3 }\end{array}$ & $\begin{array}{c}\text { NAFLD } \\
\text { absent }\end{array}$ \\
\hline AGE $<50$ & 9 & 2 & 0 & 24 \\
\hline AGE $>50$ & 54 & 31 & 4 & 76 \\
\hline total & 63 & 33 & 4 & 100 \\
\hline
\end{tabular}

\begin{tabular}{|c|c|c|c|c|c|c|}
\hline Variables & $\begin{array}{c}\text { Cases } \\
\text { Overall }\end{array}$ & $\begin{array}{c}\text { Cases } \\
\text { Males }\end{array}$ & $\begin{array}{c}\text { Cases } \\
\text { Females }\end{array}$ & $\begin{array}{c}\text { Control } \\
\text { Overall }\end{array}$ & $\begin{array}{c}\text { Control } \\
\text { Males }\end{array}$ & $\begin{array}{c}\text { Control } \\
\text { Females }\end{array}$ \\
\hline AGE & 77.7 yrs & $66.49 \mathrm{yrs}$ & $62.66 \mathrm{yrs}$ & $56.33 \mathrm{yrs}$ & 56.1 & 56.25 \\
\hline CIMT Right & 0.813 & 0.77 & 0.86 & 0.591 & 0.57 & 0.62 \\
\hline CIMT left & 0.827 & 0.794 & 0.866 & 0.59 & 0.586 & 0.629 \\
\hline
\end{tabular}

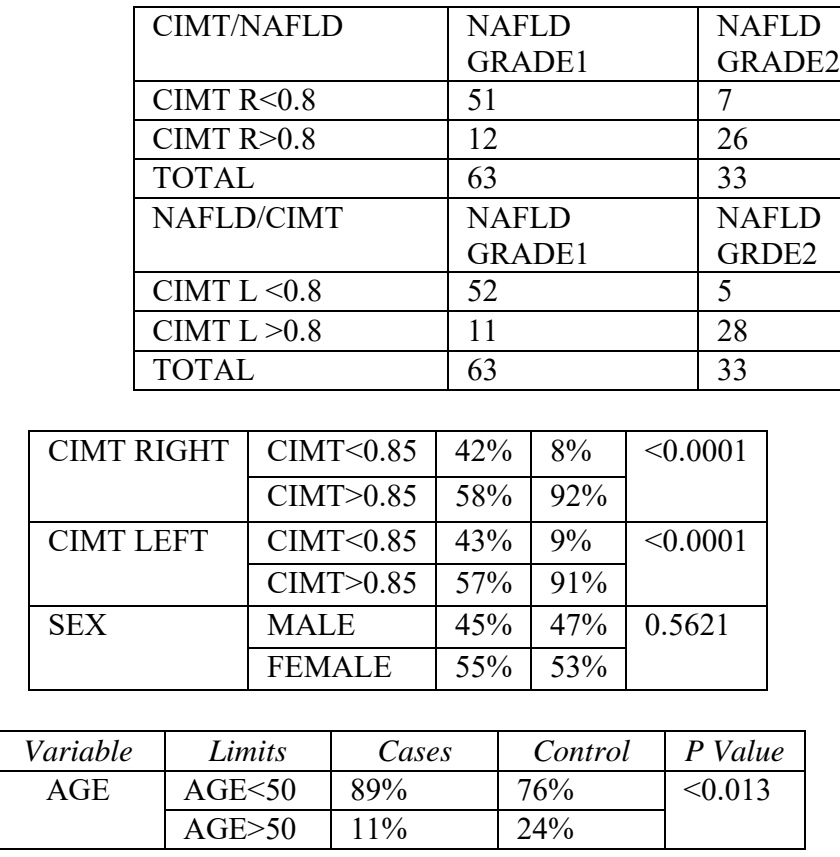

\section{Discussion}

Out of 100 cases 55 were males and 45 were females.and in control group 52were males and 48 were females.and so sex matched cases and control group were selected .There was no statistically significant relationship exists between the two groups(P VALUE-0.526)Based on the Data from other Asian countries show that the prevalence of fatty liver was higher in males being $13.3 \%$ and $2.7 \%$, respectively, in

\begin{tabular}{|l|l|l|l|}
\hline $\begin{array}{l}\text { NAFD } \\
\text { GRADE3 }\end{array}$ & NO NAFLD & TOTAL \\
\hline 0 & 92 & 150 \\
\hline 4 & 8 & 50 \\
\hline 4 & 100 & 200 \\
\hline $\begin{array}{l}\text { NAFLD } \\
\text { GRADE3 }\end{array}$ & NO NAFLD & TOTAL \\
\hline 0 & 91 & 148 \\
\hline 4 & 9 & 52 \\
\hline 4 & 100 & 200 \\
\hline
\end{tabular}

males and females in China, $21.6 \%$ and $11.2 \%$, respectively.(11)

Among cases CIMT right side was $0.813 \mathrm{~mm}$ on average and and in males was $0.772813 \mathrm{~mm}$ on average and in females was $0.86813 \mathrm{~mm}$ on average 0.591.and among control group it was $0.813 \mathrm{~mm}$ on average and in males was $0.58813 \mathrm{~mm}$ on average and in females it was $0.62813 \mathrm{~mm}$ on average which was statistically significant(P VALUE $<0.0001$ ) (P VALUE <0.0001) Targheret al ${ }^{[7]}$ studied a population of 200 diet-controlled type 2 diabetic subjects, reported that NAFLD patients had a markedly greater carotid IMT. In contrast IMT values were found to be significantly higher in diabetic patients regardless of the degree of steatosis by Cakiret $a l^{[8]}$. Similarly other two studies performed in diabetic population reported that hepatic steatosis was not associated with carotid atherosclerosis and suggested that the association of hepatic steatosis and cardiovascular disease might be just an epiphenomenon ${ }^{[9,10]}$.Targheret $a l^{[6]}$ compared carotid IMT in 85 patients with NAFLD and 160 age-, sex-, and BMImatched healthy control subjects and found that the severity

\section{Volume 5 Issue 2, February 2016}




\section{International Journal of Science and Research (IJSR) \\ ISSN (Online): 2319-7064 \\ Index Copernicus Value (2013): 6.14 | Impact Factor (2014): 5.611}

of liver histopathology in NAFLD patients was strongly associated with early carotid atherosclerosis.. Also Kucukazmanet $a l^{[15]}$ in a recent study showed a significant positive correlation between ultrasonographic steatosis grade and mean IMT $(r=0.376, P<0.001)$.

\section{Conclusion}

Herewith the end of our study it is concluded that NAFLD along with its grades is significantly positively correlated with CIMT on both sides ( $\mathrm{P}$ value $<0.0001$ ). and with age ( $\mathrm{P}$ value $<0.013$ ).NAFLD is not related to sex ( $P$ value 0.56 ).so it is concluded that NAFLD is correlated with many of the markers for cardiovascular risksindependently of classical risk factors, insulin resistance, and the presence of metabolic syndrome, suggesting that the severity of liver damage could play a role in atherosclerosis development The prevalence of NAFLD in subjects with MetS is increased four-fold compared with those without the disease and $30 \%$ of NAFLD subjects have MetS. Despite MetS itself conferring an approximate doubling of $\mathrm{CV}$ mortality risk, 15 there is still abundant evidence linking NAFLD to increased CV disease risk over and above that associated with the MetS criteria, suggesting that NAFLD per se contributes to atherosclerosis.

\section{References}

[1] Brea A, Mosquera D, Martin E, et al. Nonalcoholic fatty liver disease is associated with carotid atherosclerosis: A case-control study. ArteriosclerThrombVascBiol 2005; 25(5): 1045-1050.

[2] Acikel M, Sunay S, Koplay M, et al. Evaluation of ultrasonographic fatty liver and severity of coronary atherosclerosis, and obesity in patients undergoing coronary angiography. AnadoluKardiyolDerg 2009; 9(4): 273-9.

[3] Younossi Z, Diehl AM, Ong JP. Nonalcoholic fatty liver disease: An agenda for clinical research. Hepatology 2002; 35(4): 746-752.

[4] Kim HC, Kim DJ, Huh KB. Association between nonalcoholic fatty liver disease and carotid intimamedia thickness according to the presence of metabolic syndrome. Atherosclerosis 2009; 204(2): 521-5.

[5] Joy D, Thava VR, Scott BB. Diagnosis of fatty liver disease: Is biopsy necessary? Eur J GastroenterolHepatol 2003; 15(5): 539-43.

[6] sleislenger textbook of gastrointestinal diseases

[7] Sun L, Lu S. Association between non-alcoholic fatty liver disease and coronary artery disease severity. Chin Med J. 2011;124:867-72. [ $\underline{\text { Links }] ~}$

[8] Alper AT, Hasdemir H, Sahin S, Onturk E, Akyol A, Nurkalem $Z$, et al. The relationship between nonalcoholic fatty liver disease and the severity of coronary artery disease in patients with metabolic syndrome. Turk Kardiyol Dern Ars. 2008;36:376-81. [ Links

[9] Acikel M, Sunay S, Koplay M, Gundogdu F, Karakelleoglu S. Evaluation of ultrasonographic fatty liver and severity of coronary atherosclerosis, and obesity in patients undergoing coronary angiography. AnadoluKardiyolDerg. 2009;9:273-9. [ L Links ]

[10]Jung DH, Lee YJ, Ahn HY, Shim JY, Lee HR. Relationship of hepatic steatosis and alanine aminotransferase with coronary calcification. ClinChem Lab Med. 2010;48:1829-34. [ L Links ]

[11] Duseja A, Das A, Das R. The clinicopathological profile of Indian patients with nonalcoholic fatty liver disease (NAFLD) is different from that in the West. Dig Dis Sci 2007; 52:2368-74

[12] mohammadafsini et al international journal of general medicine orrelation of NAFLD with atherosclerotic risk factors dovepress. Afshin Mohammadi1 Ali Bazazi2 Mohammad Ghasemi-rad2 1Department of radiology, Urmia University of Medical sciences, Urmia, WestAzerbaijan, Iran; 2student research committee, Urmia University of Medical sciences, Urmia, WestAzerbaijan, Iran

[13] Targher G, Day CP, Bonora E. Risk of cardiovascular disease in patients with nonalcoholic fatty liver disease. N Engl J Med. 2010;363(14): 1341-1350.

[14] Chitturi S, Farrell GC, Hashimoto E, Saibara T, Lau GK, Sollano JD. Non-alcoholic fatty liver disease in the Asia-Pacific region: definitions and overview of proposed guidelines. J GastroenterolHepatol. 2007; 22(6):778-54).

[15] Kim D, Choi SY, Park EH, Lee W, Kang JH, Kim W, et al. Nonalcoholic fatty liver disease is associated with coronary artery calcification. Hepatology. 2012;56:60513 